\title{
Association between anorexia and hyposalivation in community-dwelling older adults in Japan: a 6-year longitudinal study
}

Yuki Ohara ${ }^{1 *}$ (D), Hisashi Kawai ${ }^{2}$, Maki Shirobe ${ }^{3}$, Keiko Motokawa ${ }^{1}$, Yoshinori Fujiwara ${ }^{4}$, Hunkyung Kim', Kazushige Ihara ${ }^{5}$, Shuichi Obuchi ${ }^{2}$, Ayako Edahiro ${ }^{1}$, Masanori Iwasaki ${ }^{1}$, Yutaka Watanabe ${ }^{6}$ and Hirohiko Hirano ${ }^{1}$

\begin{abstract}
Background: Hyposalivation is associated with the nutritional status. Anorexia of ageing, defined as an age-related decrease in appetite and food intake, presents even in healthy adults and is considered an independent predictor of malnutrition, frailty, and mortality. However, the relationship between anorexia and hyposalivation of ageing is unclear. Thus, the present longitudinal study aimed to investigate the incidence of hyposalivation and its relationship with anorexia in community-dwelling older people in Japan.

Methods: The study population comprised 220 individuals (80 men and 140 women) aged 65-86 years at baseline. The participants underwent comprehensive health check-ups, including dental examinations and anthropometry, and face-to-face interviews in 2013 and 2019. Hyposalivation was determined on the basis of the unstimulated salivary flow rate measured using the modified cotton roll method. Anorexia was defined as a score of $\leq 29$ in the Japanese version of the Council on Nutrition Appetite Questionnaire. Logistic regression analyses were used to test whether the presence of anorexia at baseline was an independent predictor of hyposalivation.
\end{abstract}

Results: Hyposalivation developed at a rate of 19.5\% during the 6-year observation period. Anorexia was observed in 95 (43.2\%) participants at baseline. After adjusting for potential confounding factors, anorexia (adjusted odds ratio $[A O R], 2.65 ; 95 \%$ confidence interval $[\mathrm{Cl}], 1.26-5.57)$ and polypharmacy (AOR, 3.29; $\mathrm{Cl}, 1.06-10.19)$ were significant predictors of hyposalivation.

Conclusion: Loss of appetite is independently correlated with and a risk factor for hyposalivation in older adults. Anorexia of ageing may have negative effects on the salivary flow rate in such settings. Salivation should be a standard feature in clinical assessments of the older adults.

Keywords: Anorexia, Oral manifestations, Appetite, Salivation, Aged

\footnotetext{
* Correspondence: yohara@tmig.or.jp

${ }^{1}$ Research Team for Promoting Independence and Mental Health, Tokyo

Metropolitan Institute of Gerontology, 35-2 Sakae-cho, Itabashi-Ku, Tokyo

173-0015, Japan

Full list of author information is available at the end of the article
}

(c) The Author(s). 2020 Open Access This article is licensed under a Creative Commons Attribution 4.0 International License, which permits use, sharing, adaptation, distribution and reproduction in any medium or format, as long as you give appropriate credit to the original author(s) and the source, provide a link to the Creative Commons licence, and indicate if changes were made. The images or other third party material in this article are included in the article's Creative Commons licence, unless indicated otherwise in a credit line to the material. If material is not included in the article's Creative Commons licence and your intended use is not permitted by statutory regulation or exceeds the permitted use, you will need to obtain permission directly from the copyright holder. To view a copy of this licence, visit http://creativecommons.org/licenses/by/4.0/. The Creative Commons Public Domain Dedication waiver (http://creativecommons.org/publicdomain/zero/1.0/) applies to the data made available in this article, unless otherwise stated in a credit line to the data. 


\section{Background}

Saliva plays an important role in the regulation of oral health because it helps in the maintenance of a neutral oral $\mathrm{pH}$ and is a reservoir of calcium and phosphate ions that are required for tooth remineralization [1]. Hyposalivation, an objective, measurable decrease in the flow of saliva, is highly prevalent in older adults [2]. In a previous study, hyposalivation was reported as a risk factor for dental caries and periodontal disease [3]. Furthermore, saliva is essential for adequate functioning of the body as it helps soften food, forms a bolus for chewing and swallowing, facilitates speech, cleans the oral tissues, and protects against tooth damage [1, 4]. Therefore, screening and early management of hyposalivation is vital for ensuring oral health, especially in individuals living in a rapidly ageing society.

Saliva contains digestive enzymes and allows for perception of the taste of foods and other substances [4]; therefore, it is also strongly associated with nutritional intake. In previous Japanese studies, older people with hyposalivation showed lower intake of certain foods, including vegetables and seafood. Hyposalivation has been associated with problems of taste perception, willingness to eat and enjoyment of meals, quality of life, and malnutrition [3, 5-7].

Anorexia of ageing is defined as an age-related decrease in appetite and food intake, and its manifestations overlap with hyposalivation. While anorexia of ageing can present in healthy older people, it is also associated with undernutrition, frailty, and mortality and, thus, can be a key indicator of the nutritional status [8]. Although Kimura et al. recently reported a significant association between anorexia and masticatory function [9], its association with hyposalivation remains unclear. Therefore, the aim of this longitudinal study was to investigate the incidence of hyposalivation and its relationship with anorexia in community-dwelling older people in Japan.

\section{Methods \\ Study population}

Details on the participant recruitment process are presented in Fig. 1. Longitudinal data were derived from the Otassha-Kenshin, a community-based cohort study conducted by the Tokyo Metropolitan Institute of Gerontology. The design and logistics of the study have been described in detail elsewhere $[10,11]$. The participants underwent a comprehensive health checkup in 2013, which was repeated 6 years later. The length of the follow-up period was determined from results of previous studies focusing on hyposalivation among community-dwelling older people $[4,12]$. The original 2013 cohort comprised 791 individuals (340 men and 451 women; mean age: 73.5 [standard deviation, 5.6] years). Individuals with hyposalivation at baseline (in 2013), those who did not participate in the follow-up survey, and those with missing data regarding the salivary flow rate in 2019 were excluded. The final study comprised 220 individuals (80 men and 140 women). This study was approved by the ethics committees of the Tokyo Metropolitan Institute of Gerontology (approval Nos. H23-1253 and R1JIN15). Written informed consent was obtained from all participants and the study was performed in accordance with the Declaration of Helsinki.

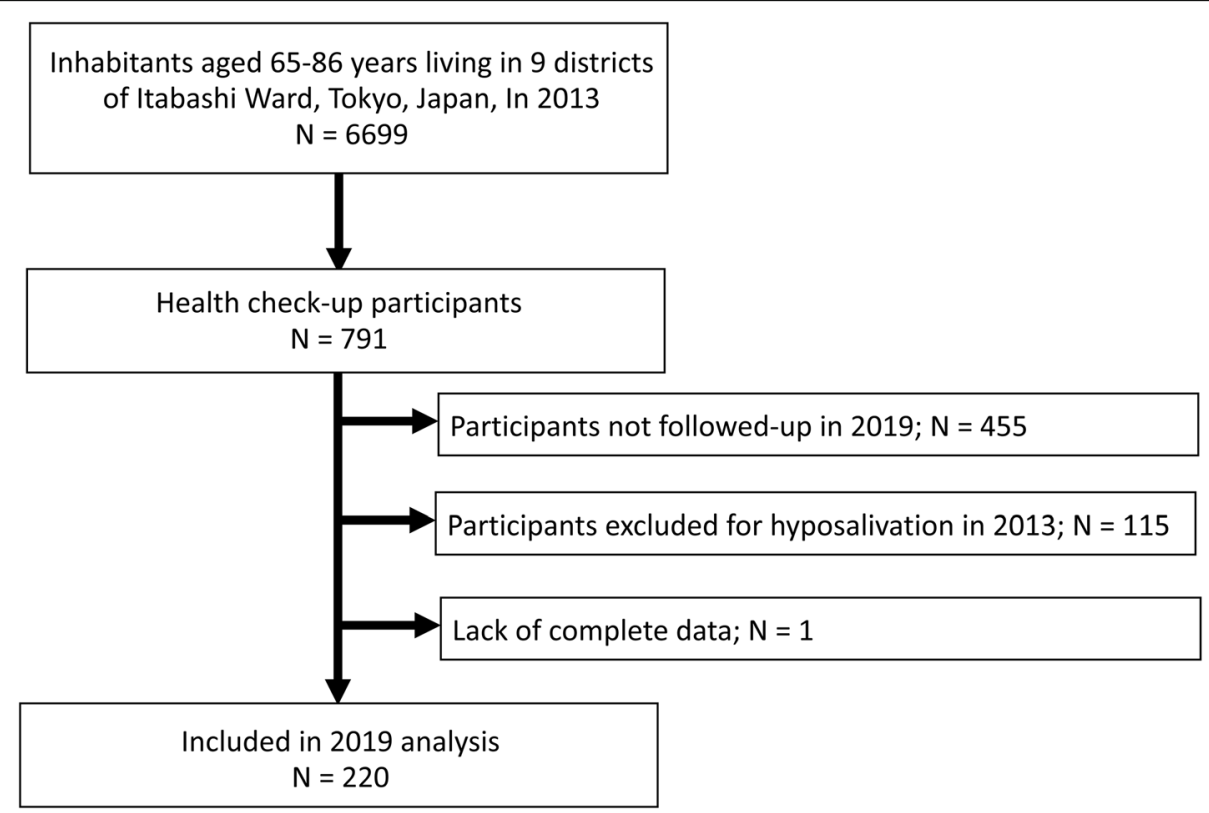

Fig. 1 Flow chart of the participant recruitment process 


\section{Assessment of the salivary flow rate}

For the determination of hyposalivation in the 2019 follow-up survey, unstimulated saliva was collected between 9:00 and 16:00 using the modified cotton roll method [13]. Participants had been instructed not to eat within $1 \mathrm{~h}$ of the assessment or drink water or other liquids within $30 \mathrm{~min}$ of the assessment. A preweighed cotton roll was placed under the tongue. The participants were instructed to close their mouth for $30 \mathrm{~s}$, and the cotton roll was subsequently removed. The amount of saliva absorbed by the cotton roll was then measured using a standardized electronic scale after calibration with copper [13, 14]. The measurement was performed once for each participant. Hyposalivation was defined as an unstimulated saliva level of $<0.1 \mathrm{~g}$, in accordance with previous studies $[13,15]$.

\section{Assessment of the oral health status}

All oral health assessments were performed by two dentists and 10 dental hygienists who had attended a 2-h training session. The training manual included an overview of the study and appropriate examination and data collection methods, and it had been constructed by the authors for the survey. For assessment of the interexaminer agreement, standardized evaluation criteria were developed by the first author, and the examiners were trained using volunteer participants until agreement with the criteria was achieved.

All assessments were conducted in a multipurpose room within our institute, with participants sitting on a chair with their head upright. The number of teeth present was recorded under appropriate illumination using artificial light. As severely decayed teeth, teeth with pulp decay, and tooth stumps are not useful for mastication, they were excluded from the count [16]. Occlusal force was measured by placing a horseshoeshaped pressure-sensitive film (Dental Prescale $50 \mathrm{H}^{\circ}$ ) between the teeth, and participants were instructed to bite it with maximum force. Subsequently, the film was analyzed with a computerized measurement system (Dental Prescale Occluzer ${ }^{\circ}$ ) that determined the maximum occlusal force [17]. The repetitive saliva swallowing test (RSST), which assesses swallowing function, was administered and involved measurement of the frequency with which patients swallowed their saliva over a 30 -s period [18]. The presence of xerostomia - the subjective feeling of oral dryness-was assessed with the dichotomous question "Does your mouth feel dry?", based on the Kihon Checklist prepared by the Japanese Ministry of Health and Welfare $[15,19]$.

\section{Appetite questionnaire}

The participants' appetite was measured in both 2013 and 2019 using a validated Japanese version of the
Council on Nutrition Appetite Questionnaire (CNAQ-J) [20]. The CNAQ-J comprises eight questions on the following items: appetite, feeling full, feeling hungry, food tastes, food tastes compared to those experienced when younger, meal frequency per day, feeling sick or nauseated when eating, and usual mood. The participants were requested to respond to each question on a 5-point Likert scale (1-5). The total score in the CNAQ-J ranges from 8 to 40 points, with lower scores indicating appetite deterioration. The reliability and validity of the CNAQ-J have been established previously [20]. Anorexia was defined as a CNAQ-J score of $\leq 29$ [21].

\section{Covariates}

We considered factors previously reported to be associated with salivary secretion and anorexia as covariates [1-9, 12, 14, 15]. Information on the participants' age, sex, smoking status, and frequency of going out was collected in 2013 using a self-administered questionnaire. The frequency of going out, included as an evaluation of the participants' level of physical activity, was assessed with the dichotomous question "Do you go out at least once a week?" [19]. In addition, trained nurses interviewed the participants regarding comorbidities and the intake of prescription medications. The use of five or more prescribed medications was defined as polypharmacy. Depressive state was evaluated by the selfadministered Zung Self-Rating Depression Scale (SDS) [22]. Body composition was evaluated using the body mass index (BMI), calculated as the body weight divided by the height squared. Blood samples were drawn for the measurement of serum albumin levels.

\section{Statistical analyses}

Statistical analyses were performed using IBM SPSS Statistics, version 25.0. The level of significance was set at $\alpha=0.05$. Participants were classified according to the presence of hyposalivation at the 6-year follow-up, and their characteristics were compared with those observed at baseline. Continuous variables were tested for normality, and Student's t-test or the Mann-Whitney U test was used for between-group comparisons. Categorical variables were compared using chi-squared or Fisher's exact tests, as appropriate. The Wilcoxon signed-rank test was used to compare changes in CNAQ-J scores between the baseline and follow-up periods. For examination of the association between anorexia and hyposalivation at baseline, logistic regression analysis with forced covariate entry was performed. Baseline variables were included as covariates if they had a $p$-value of $<0.25$ in bivariate analyses. Although age and sex had nonsignificant $p$-values in the bivariate analyses, they were forced into the models. To avoid the effects of multicollinearity, 
we confirmed that none of the potential covariates had an intercorrelation coefficient of $>0.8$.

\section{Results}

Table 1 summarizes the participants' baseline characteristics, grouped by their hyposalivation status in 2019. Over the 6-year follow-up period, $19.5 \%$ participants developed hyposalivation; no significant differences were observed with respect to sex or age. The incidence of anorexia at baseline in the hyposalivation group was $60.5 \%$, which was significantly higher than that observed in the non-hyposalivation group (39.0\%; $p=0.001)$. No significant differences were observed between the groups in baseline serum albumin or BMI levels. Similarly, in terms of oral health status at baseline, there were no significant differences between the groups in the number of teeth present, occlusal force, or swallowing function. The prevalence of xerostomia was $23.1 \%$; the difference between the groups was not significant.

Table 2 shows the changes in participants' appetite over the 6-year follow-up period. Neither group showed significant appetite-related changes as measured by the CNAQ-J scores over the 6-year observational period.

Table 3 summarizes the results of multiple logistic regression analysis of the relationship between anorexia and hyposalivation. After adjusting for potential confounding factors, anorexia was a significant predictor of hyposalivation (adjusted odds ratio [AOR], 2.65; 95\% confidence interval $[\mathrm{CI}], 1.26-5.57)$, as was polypharmacy (AOR, 3.29; CI, 1.06-10.19).

\section{Discussion}

To our knowledge, this longitudinal study is the first to examine the incidence of hyposalivation using unstimulated salivary flow measurements, and to report the association between anorexia and hyposalivation in older community-dwelling people. Several longitudinal studies have investigated the factors associated with "dry mouth" based on the presence of xerostomia, which is a subjective complaint related to salivary flow and for which data collection is simpler than for hyposalivation [23-26]. Consistent with previous studies, we did not find a significant association between hyposalivation and xerostomia [2, 15, 27], implying that they are measuring different aspects of salivary secretion. Although hyposalivation appears to be a more rigorous assessment of agerelated changes in salivary secretion, few longitudinal studies have investigated its incidence in older community-dwelling adults $[2,12]$.

In the present study, hyposalivation developed in nearly $20 \%$ of a relatively healthy older persons. Since saliva not only preserves the health status of the oral

Table 1 Baseline characteristics of the participants

\begin{tabular}{|c|c|c|c|c|c|c|c|}
\hline & \multicolumn{2}{|c|}{$\begin{array}{l}\text { Total } \\
N=220\end{array}$} & \multicolumn{2}{|c|}{$\begin{array}{l}\text { No Hyposalivation } \\
N=177(80.5 \%)\end{array}$} & \multicolumn{2}{|c|}{$\begin{array}{l}\text { Hyposalivation } \\
N=43(19.5 \%)\end{array}$} & $p$-value ${ }^{a}$ \\
\hline \multicolumn{8}{|l|}{ Demographic and general health status } \\
\hline Age, years, median (IQR) & 72 & $(69-76)$ & 72 & $(69-76)$ & 72 & $(69-76)$ & $.941^{\mathrm{b}}$ \\
\hline Sex, female, n (\%) & 140 & $(63.6)$ & 111 & $(62.7)$ & 29 & $(67.4)$ & $.563^{c}$ \\
\hline Body mass index, $\mathrm{kg} / \mathrm{m}^{2}$, median (IQR) & 22.5 & $(20.8-24.9)$ & 22.4 & $(20.8-24.8)$ & 22.6 & $(20.9-25.6)$ & $.552^{\mathrm{b}}$ \\
\hline Anorexia, n (\%) & 95 & $(43.2)$ & 69 & $(39.0)$ & 26 & $(60.5)$ & $.001^{c}$ \\
\hline Serum albumin, mg/dL, median (IQR) & 4.3 & $(4.2-4.4)$ & 4.3 & $(4.2-4.4)$ & 4.3 & $(4.2-4.5)$ & $.556^{\mathrm{b}}$ \\
\hline SDS, point, median (IQR) & 28 & $(24-32)$ & 27 & $(24-31)$ & 30 & $(24-35)$ & $.101^{\mathrm{b}}$ \\
\hline Current smoker, n (\%) & 23 & $(10.5)$ & 18 & $(10.2)$ & 5 & $(11.6)$ & $.779^{\mathrm{d}}$ \\
\hline Number of comorbidities, median (IQR) & 1 & $(0-2)$ & 1 & $(0-2)$ & 1 & $(0-2)$ & $.937^{\mathrm{b}}$ \\
\hline Polypharmacy, n (\%) & 19 & (8.6) & 12 & $(6.8)$ & 7 & $(16.3)$ & $.066^{\mathrm{c}}$ \\
\hline Going out at least once a week, n (\%) & 217 & $(98.6)$ & 175 & $(98.9)$ & 41 & $(95.3)$ & $.121^{d}$ \\
\hline \multicolumn{8}{|l|}{ Oral health status } \\
\hline Number of present teeth, median (IQR) & 24 & $(18-27)$ & 24 & $(18-27)$ & 25 & $(19-27)$ & $.882^{\mathrm{b}}$ \\
\hline Occlusal force, N, median (IQR) & 404.0 & $(223.2-568.3)$ & 404.2 & $(231.9-570.9)$ & 386.8 & $(150.6-560.6)$ & $.350^{\mathrm{b}}$ \\
\hline RSST (times/30 s), median (IQR) & 4.0 & $(3.0-5.0)$ & 4.0 & $(3.0-5.0)$ & 4.0 & $(3.0-5.0)$ & $.343^{\mathrm{b}}$ \\
\hline Xerostomia, n (\%) & 51 & $(23.2)$ & 38 & (21.5) & 12 & $(27.9)$ & $.366^{c}$ \\
\hline
\end{tabular}

Values are median (interquartile range) for continuous variables or $n$ (\%) for categorial variables. SDS, Zung Self-Rating Depression Scale; RSST, repetitive saliva swallowing test; IQR, interquartile range

Bold text indicates statistically significant associations $(p<0.05)$

${ }^{a} p$-value for the comparison between groups

${ }^{b}$ Mann-Whitney $U$ test

c chi-square test

${ }^{d}$ Fisher's exact test 
Table 2 Changes in appetite during the 6-year follow-up and comparison between the non-hyposalivation and hyposalivation groups

\begin{tabular}{|c|c|c|c|c|c|}
\hline & $\begin{array}{l}\text { Non-hyposalivation } \\
N=177\end{array}$ & $p$-value ${ }^{a}$ & $\begin{array}{l}\text { Hyposalivation } \\
\mathrm{N}=43\end{array}$ & $p$-value ${ }^{a}$ & $p$-value \\
\hline \multicolumn{6}{|c|}{ CNAQ-J, points, median (IQR) } \\
\hline Baseline & $30(29-32)$ & $0.615^{c}$ & $29(28-30)$ & $0.104^{c}$ & $0.007^{d}$ \\
\hline Follow-up & $30(28-32)$ & & $29(27-30)$ & & $0.001^{d}$ \\
\hline
\end{tabular}

CNAQ-J, Japanese version of the Council on Nutrition Appetite Questionnaire; IQR, interquartile range

a $p$-value for the comparison between baseline and follow-up period

${ }^{\mathrm{b}} p$-value for the comparison between the non-hyposalivation and hyposalivation groups

c Wilcoxon signed-rank test

${ }^{\mathrm{d}}$ Mann-Whitney $\mathrm{U}$ test

cavity but is also involved in the maintenance of general health status $[3,5,7,28]$, these findings highlight the importance of early screening and management in older adults. Both xerostomia and hyposalivation are considered to have negative impacts on the oral cavity [3, 29], and the assessment of both conditions is important in clinical settings. Our assessment of xerostomia was based on a dichotomous question; future studies should consider the use of Likert-like scales for a more precise assessment of the degree of dryness.

The findings of the present study suggest that anorexia of ageing influenced the incidence of hyposalivation even after adjustment for age, sex, medication, and psychological status $[3,5,12,15]$. Neither the hyposalivation group nor the non-hyposalivation group showed significant appetite-related changes, as measured by the CNAQ-J scores, over the 6-year observational period. However, a relationship between the two was observed in the longitudinal analyses. Thus, these results support an independent effect of baseline anorexia on the development of hyposalivation.

The observed association between hyposalivation and medication intake was consistent with the findings of previous studies $[15,30,31]$. Previous studies have reported that both anorexia of ageing and hyposalivation are associated with psychological factors [2, 30, 32]. However, in our study, decreased appetite was an independent risk factor for hyposalivation during the 6-year period, even after controlling for the effects of depressive symptoms. Anorexia of ageing is a factor that contributes to undernutrition and adverse health outcomes; therefore, interventions aimed at improving appetite should be implemented.

The primary triggers of appetite are smell and taste, and the latter greatly affects the rate of salivary secretion [8]. Salivary function is induced by mastication and gustatory stimuli [29]. The taste pathway is activated by impulses from the facial, glossopharyngeal, and vagal nerves, which have ipsilateral connections to the salivatory centers in the brainstem [29].

Generally, there is a difference between the unstimulated saliva secreted on autonomic stimulation and stimulated saliva secreted during chewing. Unstimulated saliva contains several tasting compounds, and these compounds may constantly stimulate the taste receptors located on the tongue [33]. One hypothesis that may explain our results is that the decrease in appetite induced by taste perceptions affects the amount of unstimulated saliva, and that measures aimed at improving patients' drive to eat and enjoyment of meals may lead to an increased salivary flow rate.

The major salivary glands secrete saliva for the lubrication and protection of the oral cavity in response to mucosal dryness as well as low-grade mechanical stimulation associated with lip and tongue movements [31]. Wang et al. reported that frequent gum-chewing is associated with unstimulated salivary flow [34]; thus, habitual oral movements may promote not only the rate of stimulated

Table 3 Unadjusted and adjusted logistic regression models for related covariates and hyposalivation

\begin{tabular}{|c|c|c|c|c|c|c|}
\hline & \multicolumn{3}{|c|}{ Unadjusted logistic regression } & \multicolumn{3}{|c|}{ Adjusted logistic regression } \\
\hline & OR & $95 \% \mathrm{Cl}$ & $p$-value & $\begin{array}{l}\text { Adjusted } \\
\text { OR }\end{array}$ & $95 \% \mathrm{Cl}$ & $p$-value \\
\hline Anorexia (for presence) & 2.39 & $1.21-4.73$ & 0.012 & 2.65 & $1.26-5.57$ & 0.010 \\
\hline Age, one increment & 0.99 & $0.92-1.06$ & 0.712 & 0.95 & $0.88-1.02$ & 0.184 \\
\hline Sex $($ male $=0$, female $=1)$ & 1.23 & $0.61-2.50$ & 0.563 & 1.08 & $0.51-2.29$ & 0.834 \\
\hline SDS, one increment & 1.04 & $0.99-1.08$ & 0.093 & 1.01 & $0.96-1.06$ & 0.803 \\
\hline Polypharmacy (for presence) & 2.67 & $0.98-7.26$ & 0.054 & 3.29 & $1.06-10.19$ & 0.039 \\
\hline Going out at least one a week (for yes) & 4.27 & $0.58-31.20$ & 0.153 & 5.05 & $0.62-40.78$ & 0.129 \\
\hline
\end{tabular}


saliva secretion but also that of unstimulated saliva secretion.

Decreased appetite, starting with an inability to perceive taste, and reduced daily oral movements are associated with hyposalivation [4, 29, 34]. The mechanism underlying the association between anorexia and hyposalivation was not evaluated in detail in this study because appetite was evaluated using a self-administered questionnaire. Validation with other investigative modalities, such as gustometry, is necessary in the future. Previous studies have reported that the nutritional status may also affect salivation [5, 6]; however, there were no significant associations with nutritional indices such as BMI and serum albumin level in the present study. Because this study included relatively healthy community-dwelling older adults, a relatively high BMI and serum albumin level would have been common. Further investigations using specific nutritional assessment indicators such as nutritional intake are warranted.

The limitations of this study should be recognized for accurate interpretation of the results. First, the sample comprised independently living individuals who volunteered to participate in the health examination; therefore, they likely represented a healthier portion of the general older adults. Second, over half the original participants were lost to follow-up over the 6-year period. Although this may be attributed to several factors, such as the requirement for long-term care and relocation, it has not been analyzed in detail. Third, there were inconsistencies in the definition of hyposalivation among studies. We measured the participants' unstimulated salivary flow rates using the cotton roll method in order to enable noninvasive data collection within a short time period [14]. In the future, it is advisable to evaluate both stimulated and unstimulated salivary flow rates in the same participants, and to develop a reliable comparison scale that can be applied clinically and in research. Finally, although salivary flow rates are known to vary during the day $[35,36]$, we were unable to unify the actual time of saliva assessments for each participant during the baseline and follow-up evaluations.

In conclusion, we detected the development of hyposalivation in $19.5 \%$ individuals in a cohort of older adults over a 6-year period. Further, we found that anorexia was an independent risk factor for hyposalivation. To our knowledge, this study is the first to show an effect of decreased appetite on hyposalivation. These results can be used for the development of screening or treatment protocols aimed at reducing the incidence of nutritionrelated frailty in older adults.

\section{Abbreviations}

OR: Odds ratio; Cl: Confidence interval; RSST: Repetitive saliva swallowing test; CNAQ-J: Japanese Version of the Council on Nutrition Appetite Questionnaire; SDS: Zung Self-Rating Depression Scale

\section{Acknowledgments}

The authors acknowledge Editage for English language editing.

\section{Authors' contributions}

YO, HK, and MS conceptualized and designed the study. YO, HK, MS, KM, YF, $\mathrm{HK}, \mathrm{Kl}, \mathrm{SO}$, and $\mathrm{YW}$ collected the data. $\mathrm{YO}, \mathrm{MI}$, and $\mathrm{YW}$ conducted the analysis and interpreted the data. YO drafted the initial manuscript. HK, MS, $\mathrm{KM}, \mathrm{YF}, \mathrm{HK}, \mathrm{Kl}, \mathrm{SO}, \mathrm{AE}, \mathrm{MI}, \mathrm{YW}$, and $\mathrm{HH}$ reviewed and revised the manuscript critically for important intellectual content. All authors approved the final manuscript as submitted.

\section{Funding}

This work was supported by JSPS KAKENHI [grant number 18 K09874, 18 K18462, 19 K11327, and 16 K01853], Health and Labour Sciences Research Grants from the Ministry of Health, Labour and Welfare of Japan, (H23-ChojuIppan-001 and 002, H25-Choju-lppan-005), and Research Funding for Longevity Sciences from the National Center for Geriatrics and Gerontology, Japan [grant numbers 28-30 and 29-42]. The funding body did not participate in the design of the study, participants recruitment, data collections, analysis and interpretation of data and in writing the manuscript.

\section{Availability of data and materials}

The data that support the findings of this study are available from OtasshaKenshin Study, but restrictions apply to the availability of these data, which were used under license for the current study and, therefore, are not publicly available.

\section{Ethics approval and consent to participate}

This study was approved by the ethics committees of the Tokyo Metropolitan Institute of Gerontology (approval Nos. H23-1253 and R1JIN15). Written informed consent was obtained from all participants and the study was performed in accordance with the Declaration of Helsinki.

\section{Consent for publication}

Not applicable.

\section{Competing interests}

The authors declare they have no competing interests.

\section{Author details}

${ }^{1}$ Research Team for Promoting Independence and Mental Health, Tokyo Metropolitan Institute of Gerontology, 35-2 Sakae-cho, Itabashi-Ku, Tokyo 173-0015, Japan. ${ }^{2}$ Research Team for Human Care, Tokyo Metropolitan Institute of Gerontology, Tokyo, Japan. ${ }^{3}$ The Tokyo Metropolitan Support Center for Preventative Long-term and Frail Elderly Care, Tokyo Metropolitan Institute of Gerontology, Tokyo, Japan. ${ }^{4}$ Research Team for Social Participation and Community Health, Tokyo Metropolitan Institute of Gerontology, Tokyo, Japan. ${ }^{5}$ Department of Social Medicine, Hirosaki University Graduate School of Medicine, Aomori, Japan. ${ }^{6}$ Department of Oral Health Science, Gerodontology, Faculty of Dental Medicine, Hokkaido University, Sapporo, Japan

Received: 5 September 2020 Accepted: 17 November 2020 Published online: 25 November 2020

\section{References}

1. Gupta A, Epstein JB, Sroussi H. Hyposalivation in elderly patients. J Can Dent Assoc. 2006;72:841-6.

2. Samnieng $P$, Ueno M, Shinada K, Zaitsu T, Wright FAC, Kawaguchi $Y$. Association of hyposalivation with oral function, nutrition and oral health in community-dwelling elderly Thai. Community Dent Health. 2012;29:117-23.

3. Mese H, Matsuo R. Salivary secretion, taste and hyposalivation. J Oral Rehabil. 2007:34:711-23.

4. Iwasaki M, Borgnakke WS, Yoshihara A, Ito K, Ogawa H, Nohno K, et al. Hyposalivation and 10-year all-cause mortality in an elderly Japanese population. Gerodontology. 2018;35:87-94.

5. Iwasaki M, Yoshihara A, Ito K, Sato M, Minagawa K, Muramatsu K, et al. Hyposalivation and dietary nutrient intake among community-based older Japanese. Geriatr Gerontol Int. 2016;16:500-7.

6. Muñoz-González C, Vandenberghe-Descamps M, Feron G, Canon F, Labouré $H$, Sulmont-Rossé C. Association between salivary hypofunction and food 
consumption in the elderlies. A systematic literature review. J Nutr Health Aging. 2018;22:407-19.

7. Ikebe K, Matsuda K, Morii K, Wada M, Hazeyama T, Nokubi T, et al. Impact of dry mouth and hyposalivation on oral health-related quality of life of elderly Japanese. Oral Surg Oral Med Oral Pathol Oral Radiol Endod. 2007;103:216-22.

8. Landi F, Calvani R, Tosato M, Martone AM, Ortolani E, Savera G, et al, Anorexia of aging: risk factors, consequences, and potential treatments. Nutrients. 2016:8:69.

9. Kimura Y, Iwasaki M, Ishimoto Y, Sasiwongsaroj K, Sakamoto R, Wada T, et al. Association between anorexia and poor chewing ability among community-dwelling older adults in Japan. Geriatr Gerontol Int. 2019;19: 1290-2.

10. Sakurai R, Kawai H, Yoshida H, Fukaya T, Suzuki H, Kim H, et al. Can you ride a bicycle? The ability to ride a bicycle prevents reduced social function in older adults with mobility limitation. J Epidemiol. 2016;26:307-14.

11. Sakurai R, Suzuki H, Ogawa S, Kawai H, Yoshida H, Hirano H, et al. Fear of falling, but not gait impairment, predicts subjective memory complaints in cognitively intact older adults. Geriatr Gerontol Int. 2017;17:1125-31.

12. Takeuchi K, Furuta M, Takeshita T, Shibata Y, Shimazaki Y, Akifusa S, et al. Risk factors for reduced salivary flow rate in a Japanese population: the Hisayama study. Biomed Res Int. 2015;2015:1-7.

13. Takahashi F, Koji T, Morita O. Oral dryness examinations: use of an oral moisture checking device and a modified cotton method. Prosthdont Res Pract. 2006;5:26-30.

14. Ichikawa K, Sakuma S, Yoshihara A, Miyazaki H, Funayama S, Ito K, et al. Relationships between the amount of saliva and medications in elderly individuals. Gerodontology. 2011;28:116-20.

15. Ohara Y, Hirano H, Yoshida H, Obuchi S, Ihara K, Fujiwara Y, et al. Prevalence and factors associated with xerostomia and hyposalivation among community-dwelling older people in Japan. Gerodontology. 2016;33:20-7.

16. Fukai $\mathrm{K}$, Takiguchi T, Ando Y, Aoyama H, Miyakawa Y, Ito G, et al. Associations between functional tooth number and physical complaints of community-residing adults in a 15-year cohort study. Geriatr Gerontol Int. 2009;9:366-71

17. Hidaka O, Iwasaki M, Saito M, Morimoto T. Influence of clenching intensity on bite force balance, occlusal contact area, and average bite pressure. J Dent Res. 1999;78:1336-44.

18. Oguchi K, Saitoh E, Baba M, Kusudo S, Tanaka T, Onogi K. The repetitive saliva swallowing test (RSST) as a screening test of functional dysphagia. (2) validity of RSST. Japan J Rehabil Med. 2000;37:383-8.

19. Satake S, Senda K, Hong YJ, Miura H, Endo H, Sakurai T, et al. Validity of the Kihon checklist for assessing frailty status. Geriatr Gerontol Int. 2016;16:709-15.

20. Tokudome Y, Okumura K, Kumagai Y, Hirano H, Kim H, Morishita S, et al. Development of the Japanese version of the council on nutrition appetite questionnaire and its simplified versions, and evaluation of their reliability, validity, and reproducibility. J Epidemiol. 2017;27:524-30.

21. Wilson MMG, Thomas DR, Rubenstein LZ, Chibnall JT, Anderson S, Baxi A, et al. Appetite assessment: simple appetite questionnaire predicts weight loss in community-dwelling adults and nursing home residents. Am J Clin Nutr. 2005;82:1074-81.

22. Zung WW. A self-rating depression scale. Arch Gen Psychiatry. 1965;12:63-70.

23. Nederfors T. Xerostomia and hyposalivation. Adv Dent Res. 2000;14:48-56.

24. Enoki K, Matsuda Kl, Ikebe K, Murai S, Yoshida M, Maeda Y, et al. Influence of xerostomia on oral health-related quality of life in the elderly: a 5-year longitudinal study. Oral Surg Oral Med Oral Pathol Oral Radiol. 2014;117: $716-21$

25. Johansson AK, Johansson A, Unell L, Ekback G, Ordell S, Carlsson GE. Selfreported dry mouth in 50- to 80-year-old swedes: longitudinal and crosssectional population studies. J Oral Rehabil. 2020;47:246-54.

26. Kiesswetter E, Hengeveld LM, Keijser BJ, Volkert D, Visser M. Oral health determinants of incident malnutrition in community-dwelling older adults. J Dent. 2019:85:73-80

27. Islas-Granillo H, Borges-Yáñez A, Fernández-Barrera MÁ, Ávila-Burgos L, Patiño-Marín N, Márquez-Corona ML, et al. Relationship of hyposalivation and xerostomia in Mexican elderly with socioeconomic, sociodemographic and dental factors. Sci Rep. 2017;7:40686.

28. Dodds MWJ, Johnson DA, Yeh CK. Health benefits of saliva: a review. J Dent. 2005:33:223-33.

29. Pedersen A, Sørensen CE, Proctor GB, Carpenter GH. Salivary functions in mastication, taste and textural perception, swallowing and initial digestion. Oral Dis. 2018;24:1399-416.
30. Bergdahl M, Bergdahl J. Low unstimulated salivary flow and subjective oral dryness: association with medication, anxiety, depression, and stress. J Dent Res. 2000;79:1652-8.

31. Affoo RH, Foley N, Garrick R, Siqueira WL, Martin RE. Meta-analysis of salivary flow rates in young and older adults. J Am Geriatr Soc. 2015;63:2142-51.

32. Wysokiński A, Sobów T, Kłoszewska I, Kostka T. Mechanisms of the anorexia of aging-a review. Age (Dordr). 2015;37:9821.

33. Feron G. Unstimulated saliva: background noise in taste molecules. J Texture Stud. 2019:50:6-18.

34. Wang XP, Zhong B, Chen ZK, Stewart ME, Zhang C, Zhang K, et al. History of frequent gum chewing is associated with higher unstimulated salivary flow rate and lower caries severity in healthy Chinese adults. Caries Res. 2012;46:513-8.

35. Dawes C. Circadian rhythms in the flow rate and composition of unstimulated and stimulated human submandibular saliva. J Physiol. 1975; 244:535-48.

36. Sreebny LM. Saliva in health and disease: an appraisal and update. Int Dent J. 2000;50:140-61.

\section{Publisher's Note}

Springer Nature remains neutral with regard to jurisdictional claims in published maps and institutional affiliations.
Ready to submit your research? Choose BMC and benefit from:

- fast, convenient online submission

- thorough peer review by experienced researchers in your field

- rapid publication on acceptance

- support for research data, including large and complex data types

- gold Open Access which fosters wider collaboration and increased citations

- maximum visibility for your research: over $100 \mathrm{M}$ website views per year

At BMC, research is always in progress.

Learn more biomedcentral.com/submissions 